\title{
ON THE QUANTUM COHOMOLOGY OF SOME FANO THREEFOLDS AND A CONJECTURE OF DUBROVIN
}

\author{
GIANNI CIOLLI
}

\begin{abstract}
In the present paper the small Quantum Cohomology ring of some Fano threefolds which are obtained as one- or two-curve blow-ups from $\mathbb{P}^{3}$ or the quadric $Q^{3}$ is explicitely computed. Because of systematic usage of the associativity property of quantum product only a very small and enumerative subset of Gromov-Witten invariants is needed. Then, for these threefolds the Dubrovin conjecture on the semisimplicity of Quantum Cohomology is proven by checking the computed Quantum Cohomology rings and by showing that a smooth Fano threefold $X$ with $b_{3}(X)=0$ admits a complete exceptional set of the appropriate length.
\end{abstract}

\section{INTRODUCTION}

Throughout this paper, we call Fano threefold a smooth complex compact algebraic variety of dimension three whose anticanonical divisor is ample.

Fano threefolds have been classified by Iskovskih, Mori and Mukai 23, 24, 28, 29. in 106 deformation classes, and the second Betti number $b_{2}$ ranges from 1 to 10. According to the classification, we denote with $M_{n}^{b}$ the $n$-th element of the list of Fano threefolds $M$ having $b_{2}(M)=b$.

In the present paper we compute an explicit presentation for the small Quantum Cohomology ring of 13 Fano threefolds which can be constructed as blow-ups of $\mathbb{P}^{3}$ or $Q^{3}$ along one or two smooth rational curves. Two of them were already studied, since they can also be constructed as projective bundles; however, we include them both for the sake of completeness and because our computations are quite simple.

Theorem 1. The small Quantum Cohomology ring of the Fano threefold $M_{k}^{2}$ with $k=21,22,26,27,29,30,33$ is isomorphic to the polynomial quotient ring

$$
\mathbb{C}\left[E, H, q_{0}, q_{1}\right] /\left(f_{1}^{C}-f_{1}^{Q}, f_{2}^{C}-f_{2}^{Q}\right),
$$

where the relations are described in table 2 .

Theorem 2. The small Quantum Cohomology ring of the Fano threefold $M_{n}^{3}$ for $n=10,12,15,18,20,25$ is isomorphic to the polynomial quotient ring

$$
\mathbb{C}\left[E_{1}, E_{2}, H, q_{0}, q_{1}, q_{2}\right] /\left(f_{1}^{C}-f_{1}^{Q}, f_{2}^{C}-f_{2}^{Q}, f_{3}^{C}-f_{3}^{Q}\right),
$$

where the relations are described in table 3

In section 5 we focus on a conjecture of Dubrovin:

Conjecture (Dubrovin, [16] 4.2.2 (1); [8]). Let X be a (smooth complex compact) variety. The even Quantum Cohomology ring of $X$ is generically semisimple if and only if

2000 Mathematics Subject Classification. Primary 14N35; Secondary 14J45. 
(i) $X$ is Fano and

(ii) the bounded derived category of coherent sheaves on $X$ admits a complete exceptional set whose length equals $\sum_{q} \operatorname{dim} \mathrm{H}^{q, q}(X)$.

Dubrovin conjecture has been proved by Bayer and Manin [8] for Del Pezzo surfaces; moreover, it holds for $\mathbb{P}^{n}$, because of Bel̆linson's Theorem and well-known Quantum Cohomology computations.

We prove the conjecture for other 35 Fano threefolds:

Theorem 3. Dubrovin conjecture holds for the following 36 Fano threefolds (out of the 59 having only even cohomology):

$\mathbb{P}^{3}, Q^{3}, V_{5}, V_{22}$;

$M_{k}^{2}$ with $k=21,22,24,26,27,29,30,31,32,33,34,35,36$;

$M_{k}^{3}$ with $k=10,12,15,17,18,20,24,25,27,28,30,31$;

$\mathbb{P}^{1} \times S_{k}$ with $k=2, \ldots, 8$.

The proof follows from Theorems 1] and 2] existing computations [1, 15, 31, 33] and the following proposition.

Proposition 1. Let $X$ be a smooth Fano threefold with $b_{3}(X)=0$; then the bounded derived category of coherent sheaves on $X$ admits a complete exceptional set of length $\sum_{q} \operatorname{dim} \mathrm{H}^{q, q}(X)$.

This method can eventually lead to a complete result for all the 59 Fano threefolds having only even cohomology; on the other hand, the remaining 47 Fano threefolds, i. e. those $X$ having $b_{3}>0$, present additional difficulties.

Precisely, many of such threefolds are known not to have a complete exceptional set of the appropriate length; thus, the Dubrovin conjecture is equivalent to nonsemisimplicity of the big Quantum Cohomology ring. The computation of this latter ring is much more complicated, since it involves infinitely many GromovWitten invariants; also, the relation between the small ring and non-semisimplicity of the big ring is rather implicit, contrarily to the case of Fano threefolds having $b_{3}=0$.

Acknowledgement. I wish to thank V. Ancona for many useful discussions and for having introduced me into the present subject. I am also grateful to the Dottorato di Ricerca of the University of Florence and to the Italian MIUR Project Proprietà geometriche delle varietà reali e complesse for their financial support during the preparation of my Tesi di Dottorato, when some of the results in the present paper were obtained.

\section{Quantum Cohomology}

Let $X$ be a smooth projective $n$-dimensional variety, and let $p=b_{2}(X)$. A Quantum Cohomology ring of $X$ is a tangent space to the classical cohomology ring $\mathrm{H}^{*}(X, \mathbb{C})$ endowed with a so-called quantum multiplicative structure [25] which depends on the tangency point $\gamma \in \mathrm{H}^{*}(X, \mathbb{C})$.

All the Quantum Cohomology rings with $\gamma \in \mathrm{H}^{2}(X, \mathbb{C})$ can be described simultaneously by a parametric presentation, which depends on the $p$ parameters $q_{1}, \ldots, q_{p} \in$ $\mathbb{C} \backslash\{0\}$ and gives the so-called small Quantum Cohomology ring. In this paper we are concerned only with the small Quantum Cohomology ring of $X$, which we will denote by $\mathrm{QH}(X)$; for simplicity, in the sequel we will drop the word "small". 
Let $T_{0}=1=[X], T_{1}, \ldots, T_{m}$ be a homogeneous basis of the graded vector space $\mathrm{H}^{*}(X, \mathbb{C})$ such that $\left|T_{i}\right| \leq\left|T_{j}\right|$ if $i<j$. We denote by $\bullet$ the cohomology class of a point in $X$. Let $\hat{T}_{0}=\bullet, \ldots, \hat{T}_{m} \in \mathrm{H}^{*}(X, \mathbb{C})$ be the basis which is Poincaré dual to the $T_{i}$, that is, the basis such that $\left(T_{i} \cup \hat{T}_{j}\right)_{n}=\delta_{i j} \bullet$ for all $i, j$. Let $\mathcal{B} \subset \mathrm{H}_{2}(X, \mathbb{Z})$ denote the subset of nonzero homology classes of effective rational maps to $X$. Fix $\beta_{1}, \ldots, \beta_{p} \in \mathrm{H}_{2}(X, \mathbb{Z})$ forming a vector space basis of $\mathrm{H}_{2}(X, \mathbb{C})$ and such that any $\beta \in \mathcal{B}$ can be expressed as $\sum_{i} \beta_{i} b_{i}$ with $b_{i} \in \mathbb{N}$.

Definition. Let $n \geq 3$. The moduli space $\bar{M}_{0, n}(X, \beta)$ of genus-zero $n$-marked maps of class $\beta$ in $X$ is the moduli space of isomorphism classes of stable $n$-pointed maps $\left(f, \mathcal{C}, x_{1}, \ldots, x_{n}\right)$ such that $f_{*}[\mathcal{C}]=\beta$.

Each space $\bar{M}_{0, n}(X, \beta)$ is endowed with $n$ natural evaluation maps to $X$

$$
i_{k}:\left(f, \mathcal{C}, x_{1}, \ldots, x_{n}\right) \mapsto f\left(x_{k}\right)
$$

which are involved in the following

Definition. The Gromov-Witten invariant $I_{\beta}\left(\gamma_{1}, \ldots, \gamma_{n}\right)$ is the rational number corresponding to the value of the degree-zero component of the cap product

$$
Z \cap\left(i_{1}^{*} \gamma_{1} \cup \cdots \cup i_{n}^{*} \gamma_{n}\right)
$$

where $Z \in \mathrm{CH}_{*}\left(\bar{M}_{0, n}(X, \beta)\right)$ is the virtual fundamental class.

Gromov-Witten invariants satisfy some properties [25] among which we recall the following two.

Property 1 (Divisor Axiom). Let $D$ be a divisor; then

$$
I_{\beta}\left(D, \alpha_{1}, \ldots, \alpha_{n}\right)=(\beta . D) I_{\beta}\left(\alpha_{1}, \ldots, \alpha_{n}\right) .
$$

Property 2 (Grading Axiom). Let $\alpha_{1}, \ldots, \alpha_{n}$ be homogeneous cohomology classes, i. e. $\alpha_{i} \in \mathrm{H}^{\operatorname{deg} \alpha_{i}}(M, \mathbb{C})$. If

$$
\text { virt. } \operatorname{dim} .\left(\bar{M}_{0, n}(M, \beta)\right) \neq \sum_{i} \operatorname{deg} \alpha_{i}
$$

then the Gromov-Witten invariant $I_{\beta}\left(\alpha_{1}, \ldots, \alpha_{n}\right)$ vanishes.

Remark ( $n$-pointed invariants with $n<3$ ). We can define Gromov-Witten invariants even in the case $n=1,2$ by means of the Divisor axiom: we define respectively $I_{\beta}\left(\gamma_{1}\right)$ and $I_{\beta}\left(\gamma_{1}, \gamma_{2}\right)$ as the rational numbers $x_{1}, x_{2}$ such that $I_{\beta}\left(\gamma_{1}, D_{2}, D_{3}\right)=$ $x_{1}\left(D_{2} . \beta\right)\left(D_{3} . \beta\right)$ and $I_{\beta}\left(\gamma_{1}, \gamma_{2}, D_{3}\right)=x_{2}\left(D_{3} . \beta\right)$ where $D_{1}, D_{2}$ are divisors such that $\beta . D_{i} \neq 0$. This definition is easily seen to be independent on the choice of the $D_{i}$.

Given $\alpha_{1}, \alpha_{2} \in \mathrm{H}^{*}(X, \mathbb{C})$, their quantum product is defined as

$$
\alpha_{1} * \alpha_{2}=\alpha_{1} \cup \alpha_{2}+\sum_{\beta \in \mathcal{B}} \sum_{\ell} I_{\beta}\left(\alpha_{1}, \alpha_{2}, T_{\ell}\right) \hat{T}_{\ell} q^{\beta}
$$

where $q^{\beta}:=q_{1}^{b_{1}} \cdots q_{p}^{b_{p}}$ and the $I_{\beta}\left(\gamma_{1}, \gamma_{2}, \gamma_{3}\right)$ are the three-pointed Gromov-Witten invariants, which encode enumerative information about the geometry of rational curves on $X$.

This product extends $\mathbb{C}\left[q_{1}, \ldots, q_{p}\right]$-linearly to the ring $\mathrm{H}^{*}(X, \mathbb{C})\left[q_{1}, \ldots, q_{p}\right]$. It is widely known that if $X$ is Fano, as we assume in the present paper, the sum $\sum_{\beta}$ is finite since almost all the $I_{\beta}$ vanish because of the Grading axiom (see below).

Quantum product has been proven to be associative [32, 10, 11, 27]; this property is equivalent to a large family of relations between Gromov-Witten invariants, which 
we use later to reduce the amount of the enumerative data which is essential for the determination of quantum multiplication.

These properties lead us to the following definition.

Definition. We will call a Gromov-Witten invariant $I_{\beta}\left(\alpha_{1}, \ldots, \alpha_{n}\right)$ essential if

- $\operatorname{deg} \alpha_{i}>2$ for all $i$, that is, Divisor Axiom cannot be applied any further;

- virt. $\operatorname{dim} .\left(\bar{M}_{0, n}(M, \beta)\right)=2 \sum \operatorname{deg} \alpha_{i}$, that is, it does not vanish trivially because of Grading Axiom;

- it is involved by some associativity relation in the small Quantum Cohomology ring, that is, it appears as a coefficient in some expression of type $T_{i} *\left(T_{j} * T_{k}\right)-\left(T_{i} * T_{j}\right) * T_{k}$ with $\operatorname{deg} T_{i}+\operatorname{deg} T_{j}+\operatorname{deg} T_{k} \leq 2 \operatorname{dim} X$.

From the definition it follows that essential invariants determine also non-essential ones. Thus the knowledge of all essential Gromov-Witten invariants completely determines Quantum Cohomology. Indeed, as showed in the following proposition, from the knowledge of quantum multiplication we can extract a presentation for the ring $\mathrm{QH}(X)$ as a quotient of the polynomial ring $\mathrm{H}^{*}(X, \mathbb{C})\left[q_{1}, \ldots, q_{p}\right]$.

Proposition 2 ([18, Proposition 11). Let $f_{1}, \ldots, f_{r} \in \mathbb{C}\left[x_{1}, \ldots, x_{k}\right]$ be homogeneous polynomials such that

$$
\mathrm{H}^{*}(X, \mathbb{C}) \cong \mathbb{C}\left[x_{1}, \ldots, x_{k}\right] /\left(f_{1}, \ldots, f_{r}\right)
$$

as graded rings. Let $f_{1}^{\prime}, \ldots, f_{r}^{\prime}$ be homogeneous elements in $\mathbb{C}\left[x_{1}, \ldots, x_{k}, q_{1}, \ldots, q_{p}\right]$, where for all $i \operatorname{deg} q_{i}=-K_{X} \cdot \beta_{i}$ and

- $f_{i}^{\prime}\left(x_{1}, \ldots, x_{k}, 0, \ldots, 0\right)=f_{i}\left(x_{1}, \ldots, x_{k}\right)$;

- $f_{i}^{\prime}\left(x_{1}, \ldots, x_{k}, q_{1}, \ldots, q_{p}\right)=0$ in $\mathrm{H}^{*}(X, \mathbb{C}) \otimes \mathbb{C}\left[q_{1}, \ldots, q_{p}\right]$ with the quantum product.

Then

$$
\mathrm{QH}^{*}(X, \mathbb{C}) \cong \mathbb{C}\left[x_{1}, \ldots, x_{k}, q_{1}, \ldots, q_{p}\right] /\left(f_{1}^{\prime}, \ldots, f_{r}^{\prime}\right)
$$

\section{Existing COMPUtations}

A presentation of the Quantum Cohomology ring has been computed 31, 33, 15,1 for the 21 Fano threefolds which can be constructed as $\mathbb{P}(\mathcal{E})[34$, where $\mathcal{E}$ is a rank-2 vector bundle over a surface. Also, 18 Fano threefolds have a toric representative [5. 35] and their Quantum Cohomology can be studied also with the techniques available for toric varieties $6,20,33$. These two classes overlap; their union counts 28 Fano threefolds.

Moreover, Quantum Cohomology has been computed 9] for some complete intersections in projective spaces, under some hypotheses which hold for three Fano threefolds: the quadric $M_{2}^{1}$, the cubic $M_{5}^{1}$ and $V_{5}=M_{7}^{1}$.

A few of our computations were known before. The two threefolds $M_{33}^{2}$ and $M_{25}^{3}$ are toric and could have been treated with the above-mentioned techniques. Quantum Cohomology of the threefold $M_{27}^{2}$ was already computed in 1 since $M_{27}^{2}$ can also be constructed as a projective bundle over a surface. However, we report such presentations in order to underline the analogy with the other blow-ups.

Some Gromov-Witten invariants of blow-ups along smooth curves (in particular, lemmas [6] and 9] follow also from Theorem 1.5 in 22, in which symplectic techniques are used to prove that

$$
I_{\beta}^{X}\left(\alpha_{1}, \ldots, \alpha_{m}\right)=I_{f !(\beta)}^{\tilde{X}}\left(f^{*} \alpha_{1}, \ldots, f^{*} \alpha_{m}\right)
$$


where $f !(\beta):=P f^{*} P \beta, P$ is Poincaré duality and $\beta \in \mathrm{H}_{2}(X, \mathbb{Z})$; anyway, we present our enumerative proofs, which happen to be particularly simple and clear.

We check Dubrovin conjecture by means of explicit presentations of Quantum Cohomology rings; but for some manifolds only Gromov-Witten invariants or a sketch of the computations have been done before. This is the case for the threefolds $Q^{3}, V_{5}, V_{22}$ and for trivial $\mathbb{P}^{1}$-bundles over Del Pezzo surfaces, for which we computed explicit Quantum Cohomology presentations starting from partial results in [1, 8].

Very recently, A. Bayer proved in [7] that semisimplicity of Quantum Cohomology is preserved by point blow-ups, and proposed consequently a small change to the conjecture, that is, to remove the condition of being Fano.

\section{The Blow-up of a FAno threEFold}

We recall the following description of the even cohomology ring of the blow-up of a threefold along a smooth curve (see e.g. lemma 2.11 in [23], or [19]).

Lemma 1. Let $f: \tilde{X} \rightarrow X$ be the blow-up of a smooth threefold along a smooth curve $\mathcal{C}$. Let $E \in \mathrm{H}^{2}(\tilde{X}, \mathbb{C})$ be the class of the exceptional divisor, and let $\varphi \in$ $\mathrm{H}^{4}(\tilde{X}, \mathbb{C})$ be the class of an exceptional fiber. Then

$$
\mathrm{H}^{*}(\tilde{X}, \mathbb{C}) \cong f^{*} \mathrm{H}^{*}(X, \mathbb{C}) \oplus \mathbb{C} E \oplus \mathbb{C} \varphi
$$

as vector spaces, with the multiplicative structure defined by

- $E^{2}=-f^{*}[\mathrm{C}]+c_{1} \varphi$,

- $E \cdot \varphi=-1$,

- $E . f^{*} D=(\mathcal{C} . D) \varphi$ and $\varphi . f^{*} D=0$ for all $D \in \mathrm{H}^{2}(X)$,

- $E . f^{*} C=\varphi \cdot f^{*} C=0$ for all $C \in \mathrm{H}^{4}(X)$,

where $c_{1}:=\operatorname{deg} c_{1}\left(N_{\mathcal{C}} X\right)=2 g(\mathcal{C})-2-K_{X} \cdot[\mathcal{C}]$.

Also the Quantum Cohomology structure of $\tilde{X}$ is closely related to the one in $X$. Indeed, an irreducible rational curve in $\tilde{X}$ can be either the strict transform of a rational curve in $X$ or an exceptional fiber; starting from this argument, we can build a family of finite morphisms between the various components of moduli spaces of stable maps of $X$ and $\tilde{X}$, expressing all the genus-zero Gromov-Witten invariants of $\tilde{X}$ by means of the ones of $X$, as in the example on page [

Moreover, a blow-up of the above type always gives a "standard" Gromov-Witten invariant, as stated in the next lemma; this invariant turns out to be always essential.

Lemma 2. In a threefold $\tilde{X}$ which is the blow-up along a curve, the class $F$ of an exceptional fiber is enumerative and the value of the Gromov-Witten invariant $I_{F}(\varphi)$ is -1 , where $\varphi$ is the cohomology class of an exceptional fiber.

Proof. Exceptional fibers are parameterized by the blown-up curve, and a curve in the class $F$ must be an exceptional fiber. So the dimension of the moduli space $\bar{M}_{0,3}(X, F)$ is

$$
1+3=4=3+\left(\sigma^{*}(-K)-E\right) \cdot F=3+0+1 .
$$

Let $\varrho_{i}$ be the cohomology class of the strict trasform of a line which intersects the blown-up curve transversally in $i$ points; we have $I_{F}\left(\varrho_{i}\right)=i$; since $\varrho_{0}=\varrho_{1}+\varphi$, the claim follows from (multi)linearity of Gromov-Witten invariants. 


\section{Getting information from associativity}

Only finitely many Gromov-Witten invariants are involved in the computation of all the quantum products needed in Proposition 2

Divisor and Grading axioms (see 25]) together with the fact that $b_{1}(X)=0$ (true for any Fano threefold) reduce the family of "essential" invariants to those $I_{\beta}\left(\alpha_{1}, \ldots, \alpha_{n}\right)$ such that $n \leq 3, \operatorname{deg} \alpha_{i}>2$ for all $i$ and $\sum \operatorname{deg} \alpha_{i}=-K . \beta+n$. Let's label such invariants as $x_{1}, \ldots, x_{N}$.

Consider a threefold with $b_{1}=0$, e. g. a Fano threefold; then the only nontrivial cup products are those between three divisors. This is not true for quantum product; indeed, the small Quantum Cohomology ring has infinite dimension if regarded as a $\mathbb{C}$-vector space. However, to reduce the size of the computations we considered only those associativity relations arising from triple products of divisors; they involve only a subclass of Gromov-Witten invariants, which we hope to be enough large to fulfill our purpose. This is in the spirit of the Reconstruction theorem (3.1 of [25]), which implies that the complete family of Gromov-Witten invariants is determined by the above subclass.

Consider all the possible triples $1 \leq i \leq j \leq k \leq p=b_{2}(X)$ where at least two of $i, j, k$ are distinct, and write

$$
P(i, j, k):=\left(T_{i} * T_{j}\right) * T_{k}-T_{i} *\left(T_{j} * T_{k}\right)=0 .
$$

$P$ is a polynomial in $\mathbb{C}\left[T_{1}, \ldots, T_{p}, x_{1}, \ldots, x_{N}\right]$; if we decompose it as $P(i, j, k)=$ $\sum_{I} P(i, j, k)_{I} T^{I}$, where $I=\left(i_{1}, \ldots, i_{p}\right)$ is a multi-index, $T^{I}=\prod_{s} T_{s}^{i_{s}}$ and $P(i, j, k)_{I} \in$ $\mathbb{C}\left[x_{1}, \ldots, x_{N}\right]$ we obtain a system of associativity equations

$$
\left\{\begin{array}{l}
P(i, j, k)_{I}=0 \\
\text { for all multi-indices } I \\
\text { for all non-diagonal triples }(i, j, k) \in \operatorname{Sym}^{3}\{1, \ldots, p\}
\end{array}\right.
$$

the ideal $J_{A}$ generated by such polynomials contains all the possible associativity relations among the $x_{i}$.

In general these relations do not suffice to determine all the invariants, since the affine variety $A \subset \mathbb{C}^{N}$ cut out by system (2) has positive dimension; however, by intersecting $A$ with a suitable affine variety $G$ defined by some additional geometric relations $J_{G}=\left(g_{1}, \ldots, g_{s}\right) \subset \mathbb{C}\left[x_{1}, \ldots, x_{N}\right]$, we can have that $G \cap A=\{$ one point $\}$, that is, we can determine the Quantum Cohomology of $X$ by using the minimal geometric information expressed by $G$.

To carry out this procedure, we used a specific computer program [4, 13] which computes all the equations in (21) and builds a list of all the essential invariants. Our purpose is to determine $J_{G}$, that is, a set of geometric relations which suffice to determine all the Gromov-Witten invariants of the manifold.

We begin with an empty ideal $J_{G}=(0)$. We compute a geometric relation which we don't have already, i. e. which is not contained in $J_{G}+J_{A}$ (e. g. we compute the value of some Gromov-Witten invariant not determined by associativity); we add this relation to $J_{G}$ and we check whether $J_{G}+J_{A}$ is the ideal of a single point in $\mathbb{C}^{N}$. If not, we repeat this procedure until $J_{G}+J_{A}$ is as big as desired.

In the thirteen cases that we studied (cfr. Table 1), this procedure yielded the desired results after a few steps; moreover, the standard relations coming from the blow-up construction (lemma 2) can be used as geometric relations. We refer to the following example and to 14 for further details about the computational part. 
Example. Consider the blow-up $M=M_{30}^{2}$ of $\mathbb{P}^{3}$ along a conic $\mathcal{C}$. Classical relations are $E^{2}-3 E H+2 H^{2}=0$ and $E H^{2}$; to quantize them we only need classes $\beta$ such that $3 \leq$ virt. $\operatorname{dim} . \beta \leq 6$, that is, such that $3 \geq-K_{M} \cdot \beta=(4 H-E) \cdot\left(d L_{0}+f F\right)=$ $4 d+f$, where $L_{0}=f^{*}[$ line $]$ and $F$ is the class of an exceptional fiber.

Let $f(d)$ be the minimum integer $f$ such that there exists a rational curve $Z \subset \mathbb{P}^{3}$ whose homology class is $\left[Z^{\prime}\right]=d L_{0}+f F$. Since $f(d)=-2 d$, we put $L=L_{0}-2 F$; the only $\beta$ 's satisfying $-K_{M} \cdot \beta \leq 3$ are $F, 2 F, 3 F, L$ and $L+F$.

"Exceptional" moduli spaces $\bar{M}_{0,0}(M, k F)$ are branched coverings of $\mathcal{C}$, and are easily seen to have expected dimension, as the other two. Indeed, $\bar{M}_{0,0}(M, L)$ is isomorphic to the dual of the plane spanned by $\mathcal{C}$, and $\bar{M}_{0,0}(M, L+F)$ has two components: the two-dimensional one is composed by reducible curves, and is a $2: 1$ covering of $\bar{M}_{0,0}(M, L)$, while irreducible curves lie in the three-dimensional component $Z$, because the map $\left\{(x, y) \in \mathbb{P}^{3} \times \mathfrak{C} \mid x \neq y\right\} \rightarrow Z$ sending $(x, y)$ to the strict transform of the line $\langle x, y\rangle$ is a fibration with one-dimensional fibers.

Associativity equations involve $N=14$ Gromov-Witten invariants; the affine variety $A$ has dimension three and meets in a single point the variety $G$ defined by $I_{F}(\varphi)=-1, I_{L}(\varphi, \varphi)=1$ and $I_{F+L}(\varphi, \bullet)=2$. The first invariant is standard, while the other two are easily computed considering the above description of the related moduli spaces. So Quantum Cohomology of $M$ is completely determined by associativity constraints and "simple" enumerative geometric information.

\section{Exceptional objects in the Derived Category and the Dubrovin Conjecture}

We recall some terminology and results from [30.

Definition. Let $X$ be a smooth complex projective variety. We denote with $\mathcal{D}(X)$ the bounded derived category of coherent sheaves on $X$.

Definition. An object $E$ in $\mathcal{D}(X)$ is said to be exceptional if

$$
\mathrm{R}^{i} \operatorname{Hom}(E, E)= \begin{cases}0 & \text { if } i>0 \\ \mathbb{C} & \text { if } i=0\end{cases}
$$

Definition. An exceptional set (also called a system of exceptional objects in $\mathcal{D}(X)$ of length $n+1$ is an ordered set

$$
\left(E_{0}, \cdots, E_{n}\right)
$$

such that

- all the $E_{i}$ are exceptional;

- $\mathrm{R}^{*} \operatorname{Hom}\left(E_{i}, E_{j}\right)=0$ if $i>j$ (the semiorthogonality condition).

Moreover, the set (system) is called complete (full) if it generates $\mathcal{D}(X)$ as a triangulated category.

Beylinson's theorem exhibits a complete exceptional set for the projective space $\mathbb{P}^{n}$.

Theorem 4 (Beilinson [12, 2]). Given a coherent sheaf $\mathcal{F}$ on $\mathbb{P}^{n}$, there exists a finite complex of sheaves

$$
\mathcal{G}^{\bullet}: \quad 0 \rightarrow \mathcal{G}^{-n} \rightarrow \cdots \rightarrow \mathcal{G}^{n} \rightarrow 0
$$

such that each one of the $\mathcal{G}^{q}$ is the direct sum of bundles of type $\mathcal{O}(-n), \ldots, \mathcal{O}$ and

$$
\mathrm{H}^{q}\left(\mathcal{G}^{\bullet}\right)= \begin{cases}0 & \text { if } q \neq 0 \\ \mathcal{F} & \text { if } q=0\end{cases}
$$


Indeed, in the derived category any object is equivalent to anyone of its resolutions, so these $n+1$ sheaves generate $\mathcal{D}\left(\mathbb{P}^{n}\right)$. Also, the exceptionality of the $\mathcal{O}(i)$ is an easy consequence of the fact that $\mathrm{R}^{0} F=F$ for any functor $F$ and that the $\mathcal{O}(i)$ are free, so that $\mathrm{R}^{q} \operatorname{Hom}(\mathcal{O}(i), \mathcal{O}(j))=0$ for all $q>0$ and all $i, j$. These arguments also imply semiorthogonality of the set.

Dubrovin conjecture holds for $\mathbb{P}^{n}$; indeed, $\mathrm{QH}\left(\mathbb{P}^{n}\right) \cong \mathbb{C}[H, q] /\left(H^{n+1}-q\right)$ and a corollary of Bellinson's theorem is that the length of the exceptional set is exactly

$$
\operatorname{dim} \mathrm{H}^{*}\left(\mathbb{P}^{n}, \mathbb{C}\right)=\sum_{q} \operatorname{dim} \mathrm{H}^{q, q}\left(\mathbb{P}^{n}\right)=n+1 .
$$

Conforming to Dubrovin conjecture, we will say that the appropriate length for an exceptional set of a manifold $X$ is $\sum_{q} \operatorname{dim} \mathrm{H}^{q, q}(X)$.

Starting from manifolds whose derived category has a complete exceptional set of the appropriate length, as is $\mathbb{P}^{n}$, we can construct other manifolds with the some property if we use processes which preserve both the existence of an exceptional set and the appropriatedness of its length. This is the case for some blow-ups and for all projectivizations of vector bundles, as we can deduce from the following two theorems.

Theorem 5 ([3]; Cor. 2.7 of [30]). Let $\mathcal{E} \rightarrow M$ be a vector bundle over a manifold. If $\mathcal{D}(M)$ has a complete exceptional set, then also $\mathcal{D}(\mathbb{P}(\mathcal{E})$ ) possesses a complete exceptional set.

Corollary. If $\mathcal{D}(M)$ has a complete exceptional set of the appropriate length, then also $\mathcal{D}(\mathbb{P}(\mathcal{E}))$ has a complete exceptional set of the appropriate length.

Proof. The statement about the length is a straightforward computation that can be obtained from one of the proofs of the theorem in [3, 30] and from Leray-Hirsch theorem describing the cohomology of a projective bundle.

Theorem 6 (Cor. 4.4 of [30]). Let $M$ be the blow-up of a smooth variety $X$ along a smooth subvariety $Y$. If $\mathcal{D}(X)$ has a complete exceptional set, then also $\mathcal{D}(M)$ possesses a complete exceptional set.

Corollary. Let $M$ be the blow-up of a threefold $X$ along a smooth irreducible subvariety $Y$; suppose that $\mathcal{D}(X)$ has a complete exceptional set of the appropriate length, and that $Y$ is either a point or a rational curve. Then also $\mathcal{D}(M)$ has a complete exceptional set of the appropriate length.

Proof. Again, the length of the exceptional set can be computed easily from the contents of section 4 in 30, while the appropriatedness of the length follows from lemma 1

Proof of Proposition 1. The classification of smooth Fano threefolds states that Fano threefolds having $b_{3}=0$ are $4+21+34=59$ out of 106. Indeed, they can be subdivided in the following three classes:

- the four threefolds with $b_{2}=1$, that is, $\mathbb{P}^{3}, Q^{3}, V_{5}$ and $V_{22}$;

- the twenty-one threefolds which are $\mathbb{P}^{1}$-bundles over surfaces;

- the thirty-four threefolds which are not $\mathbb{P}^{1}$-bundles over surfaces and can be obtained by blowing up another Fano threefold along a smooth subvariety $Y$ having only cohomology of even degree.

Fano threefolds with $b_{2}=1$ for which (cfr. 8) a complete exceptional set in $\mathcal{D}(X)$ is known to exist are $\mathbb{P}^{3}, Q^{3}, V_{5}$ [30] and $V_{22}$ [26, [17; they are the only ones 
with Pic $=\mathbb{Z}$ having only even cohomology. Moreover, the exceptional set has the appropriate length.

All the 21 Fano threefolds $M_{1}, \ldots, M_{21}$ which are $\mathbb{P}^{1}$-bundles over surfaces, corresponding to the 21 Fano vector bundles in [34] (we refer to that paper or to [1] for the notation), have a complete exceptional set in $\mathcal{D}\left(M_{i}\right)$ of the appropriate length.

Indeed, for all the surfaces $S$ which appear as bases of such bundles $\mathcal{D}(S)$ has a complete exceptional set of the appropriate length, since they are $\mathbb{P}^{2}, \mathbb{P}^{1} \times \mathbb{P}^{1}$ which can be thought as the projectivization of trivial vector bundles over $\mathbb{P}^{1}$ - and Del Pezzo surfaces, for whom a computation quite similar to the one in Theorem 6 can be made. Thus only a further application of the corollary of Theorem 5 proves the claim.

Finally, the corollary of Theorem $[$ shows inductively the existence of a complete exceptional set of the appropriate length in $\mathcal{D}(X)$ for any $X$ in the third class.

\section{Proofs of the main Results}

We denote by $\bullet, \varphi$ and $\varrho$ the cohomology class respectively of a point, of an exceptional fiber and of the pullback of a generic line class in $\mathbb{P}^{3}$ or $Q^{3}$; we put $L_{0}=(\varrho)_{*}$ and $F=(\varphi)_{*}$, where $(-)_{*}: \mathrm{H}^{p}(M, \mathbb{C}) \rightarrow \mathrm{H}_{p}(M, \mathbb{C})$ denotes Poincaré duality.

Proof of Theorem 1 Lemma 1 gives the classical relations.

The maximal $G$, as in section 4 is defined by the standard equation $I_{F}(\varphi)=-1$ (given by lemma 2) plus the following equations, for which we refer to the lemmas in the last section:

$$
\begin{array}{lr}
I_{L_{0}-F}(\varrho, \bullet)=\operatorname{deg} \varrho & \text { for } n=22,27,30,33 ; \\
I_{L_{0}-2 F}(\varrho, \varrho)=1 & \text { for } n=30 ; \\
I_{L_{0}}(\varrho, \bullet)=1 \text { and } I_{L_{0}-F}(\varrho, \varphi)=1 & \text { for } n=21,26,29 .
\end{array}
$$

Proof of Theorem [ As for the previous theorem, Lemma 1 gives the classical relations. Moreover, in these cases quadratic relations suffice to generate the cohomology ring, as it can be easily verified. Since all of these threefold are obtained by blowing up two disjoint curves, we have two distinct homology classes $F_{1}, F_{2}$ of exceptional fibers and consequently two standard Gromov-Witten invariants of the type described in lemma 2 which for all these varieties turn out to be independent: i. e., they cut out a 2-codimensional subvariety of $A$.

The maximal $G$ (cfr. section 4) is defined by the standard equations $I_{F_{1}}\left(\varphi_{1}\right)=$ $I_{F_{2}}\left(\varphi_{2}\right)=-1$ plus the following ones, for which we refer to the lemmas in the last section:

$$
\begin{array}{ll}
I_{L_{0}}(\varrho, \bullet)=1 & \text { for } n=10,15,20, \\
I_{L_{0}-F_{1}-F_{2}}(\bullet)=\operatorname{deg} \mathcal{C}_{2} & \text { for } n=12,18,25,
\end{array}
$$

where $\mathcal{C}_{2}$ is the smooth rational curve such that the threefold is obtained by blowing up $\mathbb{P}^{3}$ along a disjoint union (line) $\sqcup \mathcal{C}_{2}$.

Proof of Theorem 3. The existence of an exceptional set is positively answered for all these varieties by Proposition 10 Generic semisimplicity is verified with a standard commutative algebra software using the explicit presentations worked out in the present paper and in 11, 15, 31, 33, after the following lemma. 
Lemma 3. Generic semisimplicity of Quantum Cohomology is a consequence of the existence of a single semisimple point $\gamma \in \mathrm{H}^{2}(X, \mathbb{C})$. This in turn is equivalent to generic semisimplicity over the $\mathrm{H}^{2}(X, \mathbb{C})$.

To see this, consider the following diagram:

$$
\mathbb{C}^{c+p} \supset Z=V\left(f_{1}^{\prime}, \ldots, f_{k}^{\prime}\right) \stackrel{\pi}{\rightarrow}\left(\mathbb{C}^{*}\right)^{p} \stackrel{\psi}{\leftarrow} \mathrm{H}^{2}(X, \mathbb{C}),
$$

where $\pi$ is the projection forgetting the $q_{i}$ 's and $\psi\left(\gamma_{1}, \ldots, \gamma_{p}\right)=\left(\exp \left(\gamma_{1}\right), \ldots, \exp \left(\gamma_{p}\right)\right)$. Semisimplicity of the small Quantum Cohomology ring is equivalent to the fact that $\psi(S)$ is a nonempty Zariski open subset in $\left(\mathbb{C}^{*}\right)^{p}$, where $S$ is the locus of the points $\gamma \in \mathrm{H}^{2}(X, \mathbb{C})$ such that the Quantum Cohomology $\mathrm{QH}_{\gamma}(X)$ is semisimple.

Indeed, semisimple points $\gamma \in S$ correspond exactly to the points $q=\psi(\gamma) \in\left(\mathbb{C}^{*}\right)^{p}$ such that $\pi^{-1}(q)$ is a reduced zero-dimensional scheme. Moreover, the fiber $\pi^{-1}(\gamma)$ is obtained by putting $q_{i}:=\psi\left(\gamma_{i}\right) \forall i$, that is, by intersecting $Z$ with a linear variety of codimension $p$. So $\psi(S)$ is exactly the locus in $\left(\mathbb{C}^{*}\right)^{p}$ over which the fibers of $\pi$ are reduced, which is an open Zariski subset. If it is empty, then all the fibers are nonreduced, that is, $Z$ itself is not reduced and the small Quantum Cohomology is not semisimple.

Lemma 4. $I_{L_{0}-F}(\varrho, \bullet)=\operatorname{deg} \mathcal{C}$ for $X=M_{k}^{2}, k=22,27,30,33$.

Proof. Let $\ell \in \varrho$ and $x \in \bullet$ be generic representatives.

Irreducible curves $\mathcal{C}^{\prime}$ of class $L_{0}-F$ are strict transforms of lines intersecting $\mathcal{C}$ in exactly one point. The map sending $\mathrm{C}^{\prime} \in \bar{M}_{0,0}\left(X, L_{0}-F\right)$ to $\mathrm{C}^{\prime} \cap \mathcal{C}$ is a fibration over $\mathcal{C}$ with bidimensional fibers.

If $\operatorname{deg} \mathcal{C}>1$, the class $L_{0}-F$ contains also reducible curves; we want to show that these curves do not contribute to the invariant, i. e., that no line intersecting $\mathcal{C}$ with multiplicity greater than 1 can meet both $x$ and $\ell$.

Indeed, choose a generic plane $\mathbb{P}^{2}$ containing $\ell$. The projection $\pi_{x}: \mathbb{P}^{3} \rightarrow \mathbb{P}^{2}$ maps $\mathcal{C}$ into a plane rational curve $\mathcal{C}_{0}$ with $m=\frac{1}{2}(d-1)(d-2)$ nodes $x_{1}, \ldots, x_{m}$. The lines $\overline{x_{i} x}$ are exactly all the lines passing through $x$ and $\ell$ and intersecting $\mathcal{C}$ with multiplicity greater than 1 . Genericity of $x$ implies that none of these $m$ lines meets $\ell$, so that no reducible curve in class $L_{0}-F$ is involved in the computation of this Gromov-Witten invariant.

This implies both the enumerativeness of the invariant, since we have seen above that $\operatorname{dim} \bar{M}_{0,0}\left(X, L_{0}-F\right)=3$, and the possibility to compute it considering only irreducible curves, that is, counting the number of strict transforms of lines $\ell$ touching $\mathcal{C}$ in exactly one point.

The cone projecting $\mathcal{C}$ from $x$ intersects $\ell$ in $d=\operatorname{deg} \mathcal{C}$ points, which belong to the lines $\ell_{i}(i=1, \ldots, d)$ in the the cone. The strict transforms of the $\ell_{i}$ are thus all the rational curves belonging to class $L_{0}-F$ and meeting the strict transforms of $x$ and $\ell$, thus proving the claim.

Lemma 5. $I_{L_{0}-2 F}(\varrho, \varrho)=1$ for $M_{30}^{2}$.

Proof. Lines who are bisecant a given conic in $\mathbb{P}^{n}$ are parameterized by the dual of the plane spanned by the conic; thus the dimension of $\bar{M}_{0,0}\left(M_{30}^{2}, L_{0}-2 F\right)$ is $2+3=5=$ virt. $\operatorname{dim} .\left(L_{0}-2 F\right)$ and we can use the enumerative interpretation.

Let $Z$ be the plane spanned by the conic $\mathcal{C}$, and let $\ell_{1}, \ell_{2}$ be two generic lines in $\mathbb{P}^{3} ;$ let $Z \cap \ell_{i}=\left\{x_{1}\right\}$. 
The value of $I_{L_{0}-2 F}(\varrho, \varrho)$ is one, since it counts the number of lines $\ell$ included in $Z$ whose strict transform $\ell^{\prime}$ meets the strict transforms of $x_{1}$ and $x_{2}$, that is, the number of lines in a plane passing through two generic points.

Lemma 6. $I_{L_{0}}(\varrho, \bullet)=1$ for $M_{k}^{2}, k=21,26,29$.

Proof. Let $M$ be the blow-up of $Q^{3}$ along the rational curve $\mathrm{C}$.

The family of lines in $Q^{3}$ is three-dimensional; the class of the strict transform of a line $\ell$ is $L_{0}$ if and only if $\ell$ does not meet $\mathcal{C}$; it is easy to see that the subspace of reducible curves in $\bar{M}_{0,3}\left(M, L_{0}\right)$ has positive codimension.

Thus the dimension of the whole space of rational curves having class $L_{0}$ is $3+3=$ $6=\operatorname{virt} \cdot \operatorname{dim} .\left(L_{0}\right)$ and the enumerative interpretation is legitimate, that is, the invariant $I_{L_{0}}(\varrho, \bullet)$ counts the number of rational curves of class $L_{0}$ meeting the strict transforms of a generic line and a generic point in $Q^{3}$.

Consider a generic line $\ell^{\prime} \subset Q^{3}$ and a generic point $x^{\prime} \in Q^{3}$; there exists exactly one line $\ell \subset Q^{3}$ meeting both $x^{\prime}$ and $\ell^{\prime}$; moreover, the genericity of $x^{\prime}$ and $\ell^{\prime}$ implies that $\ell$ does not meet $\mathcal{C}$. Since any curve of class $L_{0}$ is the strict transform of a line $\ell$ in $Q^{3}$ (plus some exceptional fiber if $\ell$ meets $\mathcal{C}$ ) $\ell$ is the only curve of class $L_{0}$ meeting both $x^{\prime}$ and $\ell^{\prime}$, and $I_{L_{0}}(\varrho, \bullet)=1$.

Lemma 7. $I_{L_{0}-F}(\varrho, \varphi)=1$ for $M_{k}^{2}, k=21,26,29$.

Proof. A curve of class $L_{0}-F$ which meets a generic line $\varrho^{\prime}$ cannot be decomposed as $\mathcal{C}^{\prime} \sqcup \varphi^{\prime}$, since in that case $\mathcal{C}^{\prime}$ would be bisecant to $\mathcal{C}$ and thus could not meet $\varrho^{\prime}$.

An irreducible curve of class $L_{0}-F$ is the strict transform of a line meeting $\mathcal{C}$ in degree 1; the claim follows from $N_{1,1}=1$, where $N_{1,1}$ is the number of lines meeting a generic point and a generic line (see [21]).

Lemma $8\left(M_{k}^{3}\right.$ for $\left.k=12,18,25\right)$. Let $\mathcal{C}_{2} \subset \mathbb{P}^{3}$ be a smooth rational curve of degree $2 \leq d \leq 4$ which is disjoint from a line $\mathcal{C}_{1} \subset \mathbb{P}^{3}$; let $M$ be the blow-up of $\mathbb{P}^{3}$ along $\mathcal{C}_{1} \sqcup \mathcal{C}_{2}$. Then $I_{L_{0}-F_{1}-F_{2}}(\bullet)=d$.

Proof. A curve of class $\beta=L_{0}-F_{1}-F_{2}$ is the strict transform of a line meeting $\mathcal{C}_{1}$ and $\mathcal{C}_{2}$ in one point each, or the connected union of the strict transform of a line which intersects $\mathcal{C}_{1}$ in one point and $\mathcal{C}_{2}$ in a scheme of degree $k>1$ with some exceptional fibers over $\mathcal{C}_{2}$. The class $\beta$ is enumerative, since reducible curves form at most a 1-dimensional family and irreducible ones form a 2-dimensional family.

Consider the plane spanned by a generic point $x \in \mathbb{P}^{3}$ and the line $\mathcal{C}_{1}$; it intersects $\mathcal{C}_{2}$ in a zero-dimensional scheme of degree $\operatorname{deg} \mathcal{C}_{2}$, and the intersection is transverse by the genericity assumptions on $x$. This implies both that no reducible curves of class $\beta$ meet the strict transform $x^{\prime}$ of $x$, and that exactly $\operatorname{deg} \mathcal{C}_{2}$ irreducible curves of class $\beta$ meet $x^{\prime}$, which proves the lemma.

Lemma 9. $I_{L_{0}}(\varrho, \bullet)=1$ for $M_{k}^{3}$ with $k=10,15,20$.

Proof. It is easy to see that the subspace of reducible curves has positive codimension in the moduli space $\bar{M}_{0,0}\left(M, L_{0}\right)$; so

$$
\operatorname{dim} \bar{M}_{0,0}\left(M, L_{0}\right)=\operatorname{dim}\{\text { lines in the quadric }\}=3 .
$$

The invariant $I_{L_{0}}(\varrho, \bullet)$ is thus enumerative, and counts the number of curves in the class $L_{0}$ which intersects the strict transforms both of a generic point $x^{\prime}$ and a generic line $\ell^{\prime}$ in $Q^{3}$. 
Genericity assumptions imply that the unique $\ell$ meeting both $x^{\prime}$ and $\ell^{\prime}$ does not touch the blown-up locus; so the value of the invariant is $N_{1,1}=1$, as in lemma 7

TABle 1. Amount of geometric information needed in order to determine Quantum Cohomology. $L$ denotes a line, $C$ a conic, $T$ a twisted cubic and $U$ a rational quartic.

\begin{tabular}{l|rrr} 
Threefold & $N$ & $\operatorname{dim} A$ & $\operatorname{deg} A$ \\
\hline$M_{22}^{2}=$ blow-up of $\mathbb{P}^{3}$ along $U$ & 24 & 2 & 3 \\
$M_{27}^{2}=$ blow-up of $\mathbb{P}^{3}$ along $T$ & 14 & 2 & 2 \\
$M_{30}^{2}=$ blow-up of $\mathbb{P}^{3}$ along $C$ & 14 & 3 & 1 \\
$M_{33}^{2}=$ blow-up of $\mathbb{P}^{3}$ along $L$ & 10 & 2 & 1 \\
\hline$M_{21}^{2}=$ blow-up of $Q^{3}$ along $U$ & 24 & 3 & 5 \\
$M_{26}^{2}=$ blow-up of $Q^{3}$ along $T$ & 24 & 3 & 3 \\
$M_{29}^{2}=$ blow-up of $Q^{3}$ along $C$ & 14 & 3 & 2 \\
\hline$M_{12}^{3}=$ blow-up of $\mathbb{P}^{3}$ along $L \sqcup T$ & 81 & 3 & 9 \\
$M_{18}^{3}=$ blow-up of $\mathbb{P}^{3}$ along $L \sqcup C$ & 81 & 3 & 6 \\
$M_{25}^{3}=$ blow-up of $\mathbb{P}^{3}$ along $L \sqcup L$ & 52 & 3 & 3 \\
\hline$M_{10}^{3}=$ blow-up of $Q^{3}$ along $C \sqcup C$ & 81 & 3 & 23 \\
$M_{15}^{3}=$ blow-up of $Q^{3}$ along $L \sqcup C$ & 81 & 3 & 7 \\
$M_{20}^{3}=$ blow-up of $Q^{3}$ along $L \sqcup L$ & 81 & 3 & 5
\end{tabular}

\section{REFERENCES}

1. Vincenzo Ancona and Marco Maggesi, Quantum Cohomology of some Fano threefolds, to appear in Advances in Geometry, 2002.

2. Vincenzo Ancona and Giorgio Ottaviani, An introduction to the derived categories and the theorem of Beilinson, Atti Accad. Peloritana Pericolanti Cl. Sci. Fis. Mat. Natur. 67 (1989), 99-110 (1991). MR 92g:14013

3. Canonical resolutions of sheaves on Schubert and Brieskorn varieties, Complex analysis (Wuppertal, 1991), Aspects Math., no. E17, Vieweg, Braunschweig, 1991, pp. 14-19.

4. Giovanni Baldini, Gianni Ciolli, and Marco Maggesi, Algheme, a commutative algebra package for the Scheme language, Freely available at http://sourceforge.net/projects/algheme/.

5. V. V. Batyrev, Toric Fano threefolds, Izv. Akad. Nauk SSSR Ser. Mat. 45 (1981), no. 4, 704-717, 927. MR 82m:14022

6. Victor V. Batyrev, Quantum cohomology rings of toric manifolds, Astérisque (1993), no. 218, 9-34, Journées de Géométrie Algébrique d'Orsay (Orsay, 1992). MR 95b:32034

7. Arend Bayer, Semisimple Quantum Cohomology and Blow-ups, Preprint arXiv:math.AG/0403260, 2004.

8. Arend Bayer and Yuri I. Manin, (Semi)simple exercises in Quantum Cohomology, Preprint arXiv:math.AG/0103164, 2001.

9. A. Beauville, Quantum cohomology of complete intersections, Mat. Fiz. Anal. Geom. 2 (1995), no. 3-4, 384-398.

10. K. Behrend, Gromov-Witten invariants in algebraic geometry, Invent. Math. 127 (1997), no. 3, 601-617. MR 98i:14015 
TABLE 2. Relations in the small Quantum Cohomology rings of threefolds described in Theorem [1

\begin{tabular}{|c|c|c|}
\hline$X$ & $f_{i}^{C}$ & $f_{i}^{Q}$ \\
\hline \multirow[t]{2}{*}{$M_{21}^{2}$} & $E H^{2}$ & $\begin{array}{l}-8 E H q_{0}+10 H^{2} q_{0}-28 E q_{0}^{2}-6 H q_{0}^{2} \\
+8 H q_{0} q_{1}+16 q_{0}^{2} q_{1}\end{array}$ \\
\hline & $E^{2}-\frac{5}{2} E H+2 H^{2}$ & $-2 E q_{0}+3 H q_{0}+E q_{1}+2 q_{0} q_{1}$ \\
\hline \multirow[t]{2}{*}{$M_{22}^{2}$} & $E H^{2}$ & $\begin{array}{l}-6 E H q_{0}+10 H^{2} q_{0}-15 E q_{0}^{2}-6 H q_{0}^{2} \\
-4 E q_{0} q_{1}+18 H q_{0} q_{1}+4 q_{0} q_{1}^{2}\end{array}$ \\
\hline & $E^{2}-\frac{7}{2} E H+4 H^{2}$ & $-\frac{5}{2} E q_{0}+5 H q_{0}+E q_{1}+3 q_{0} q_{1}$ \\
\hline \multirow[t]{2}{*}{$M_{26}^{2}$} & $E H^{2}$ & $-4 E H q_{0}+\frac{7}{2} H^{2} q_{0}-6 E q_{0}^{2}-2 H q_{0}^{2}+6 H q_{0} q_{1}$ \\
\hline & $E^{2}-\frac{7}{3} E H+\frac{3}{2} H^{2}$ & $-\frac{5}{6} E q_{0}+\frac{5}{6} H q_{0}+E q_{1}+\frac{1}{2} q_{0} q_{1}$ \\
\hline \multirow[t]{2}{*}{$M_{27}^{2}$} & $E H^{2}$ & $-3 E q_{0}+8 H q_{0}+3 q_{0} q_{1}$ \\
\hline & $E^{2}-\frac{10}{3} E H+3 H^{2}$ & $E q_{1}+\frac{1}{3} q_{0}$ \\
\hline \multirow[t]{2}{*}{$M_{29}^{2}$} & $E H^{2}$ & $4 H q_{0}$ \\
\hline & $E^{2}-2 E H+H^{2}$ & $E q_{1}$ \\
\hline \multirow[t]{2}{*}{$M_{30}^{2}$} & $E H^{2}$ & $-2 E q_{0}+2 H q_{0}+2 q_{0} q_{1}$ \\
\hline & $E^{2}-3 E H+2 H^{2}$ & $E q_{1}$ \\
\hline \multirow[t]{2}{*}{$M_{33}^{2}$} & $E H^{2}$ & $q_{0}$ \\
\hline & $E^{2}-2 E H+H^{2}$ & $E q_{1}$ \\
\hline
\end{tabular}

11. K. Behrend and B. Fantechi, The intrinsic normal cone, Invent. Math. 128 (1997), no. 1, 45-88. MR 98e: 14022

12. A. A. Beilinson, Coherent sheaves on $\mathbf{P}^{n}$ and problems in linear algebra, Funktsional. Anal. i Prilozhen. 12 (1978), no. 3, 68-69. MR 80c:14010b

13. Gianni Ciolli, fano3folds, a software for the systematic computation of Associativity relations for Fano threefolds, can be downloaded freely at http://www.math.unifi.it/ ciolli/fano3folds, 2003.

14. On the Quantum Cohomology of some Fano threefolds and a conjecture of Dubrovin, Tesi di Dottorato, 2003.

15. L. Costa and R. M. Mirò-Roig, Quantum cohomology of projective bundles over $\mathbb{P}^{n_{1}} \times \cdots \times \mathbb{P}^{n_{s}}$, International J. of Math. 11 (2000), no. 6, 761-797.

16. Boris Dubrovin, Geometry and analytic theory of Frobenius manifolds, Proceedings of the International Congress of Mathematicians, Vol. II, Doc. Math. 1998, no. Extra Vol. II, Berlin, 1998, pp. 315-326.

17. Daniele Faenzi, Bundles over Fano Threefolds of type $V_{22}$, quaderno del Dipartimento di Matematica "Ulisse Dini", 2003.

18. W. Fulton and R. Pandharipande, Notes on stable maps and quantum cohomology, Algebraic geometry - Santa Cruz 1995, Proc. Sympos. Pure Math., vol. 62, Amer. Math. Soc., Providence, RI, 1997, pp. 45-96. MR 98m:14025

19. William Fulton, Intersection theory, Ergebnisse der Mathematik und ihrer Grenzgebiete (3) [Results in Mathematics and Related Areas (3)], vol. 2, Springer-Verlag, Berlin, 1984. MR 85k:14004 
TABLE 3. Relations in the small Quantum Cohomology rings of threefolds described in Theorem 2

\begin{tabular}{l|ll}
$X$ & $f_{i}^{C}$ & $f_{i}^{Q}$ \\
\hline$M_{10}^{3}$ & $E_{1} E_{2}$ & $-2 E_{1} q_{0}-2 E_{2} q_{0}+4 H q_{0}+4 q_{0}^{2}$ \\
& $E_{1}^{2}-2 E_{1} H+H^{2}$ & $E_{1} q_{1}+2 q_{0} q_{1}$ \\
& $E_{2}^{2}-2 E_{2} H+H^{2}$ & $E_{2} q_{2}+2 q_{0} q_{2}$ \\
\hline$M_{12}^{3}$ & $E_{1} E_{2}$ & $-2 E_{1} q_{0}-4 E_{2} q_{0}+8 H q_{0}+3 q_{0} q_{2}$ \\
& $E_{1}^{2}-2 E_{1} H+H^{2}$ & $E_{1} q_{1}+q_{0} q_{1}$ \\
& $E_{2}^{2}-\frac{10}{3} E_{2} H+3 H^{2}$ & $-\frac{1}{3} E_{1} q_{0}-\frac{2}{3} E_{2} q_{0}+\frac{4}{3} H q_{0}+\frac{1}{3} q_{0} q_{1}+E_{2} q_{2}+2 q_{0} q_{2}$ \\
\hline$M_{15}^{3}$ & $E_{1} E_{2}$ & $-2 E_{1} q_{0}-E_{2} q_{0}+2 H q_{0}$ \\
& $E_{1}^{2}-E_{1} H+\frac{1}{2} H^{2}$ & $-E_{1} q_{0}-\frac{1}{2} E_{2} q_{0}+H q_{0}+E_{1} q_{1}+q_{0} q_{1}+\frac{1}{2} q_{0} q_{2}$ \\
& $E_{2}^{2}-2 E_{2} H+H^{2}$ & $E_{2} q_{2}+q_{0} q_{2}$ \\
\hline$M_{18}^{3}$ & $E_{1} E_{2}$ & $-2 E_{2} q_{0}+2 H q_{0}+2 q_{0} q_{2}$ \\
& $E_{1}^{2}-2 E_{1} H+H^{2}$ & $E_{1} q_{1}$ \\
& $E_{2}^{2}-3 E_{2} H+2 H^{2}$ & $E_{2} q_{2}$ \\
\hline$M_{20}^{3}$ & $E_{1} E_{2}$ & $-E_{1} q_{0}-E_{2} q_{0}+H q_{0}$ \\
& $E_{1}^{2}-E_{1} H+\frac{1}{2} H^{2}$ & $-\frac{1}{2} E_{1} q_{0}-\frac{1}{2} E_{2} q_{0}+\frac{1}{2} H q_{0}+E_{1} q_{1}+\frac{1}{2} q_{0} q_{1}+\frac{1}{2} q_{0} q_{2}$ \\
& $E_{2}^{2}-E_{2} H+\frac{1}{2} H^{2}$ & $-\frac{1}{2} E_{1} q_{0}-\frac{1}{2} E_{2} q_{0}+\frac{1}{2} H q_{0}+\frac{1}{2} q_{0} q_{1}+E_{2} q_{2}+\frac{1}{2} q_{0} q_{2}$ \\
\hline$M_{25}^{3}$ & $E_{1} E_{2}$ & $q_{0}$ \\
& $E_{1}^{2}-2 E_{1} H+H^{2}$ & $E_{1} q_{1}$ \\
& $E_{2}^{2}-2 E_{2} H+H^{2}$ & $E_{2} q_{2}$
\end{tabular}

20. Alexander Givental, A mirror theorem for toric complete intersections, Topological field theory, primitive forms and related topics (Kyoto, 1996), Progr. Math., vol. 160, Birkhäuser Boston, Boston, MA, 1998, pp. 141-175. MR 2000a:14063

21. L. Göttsche and R. Pandharipande, The Quantum Cohomology of blow-ups of $\mathbb{P}^{2}$ and enumerative geometry, J. Differential Geom. 48 (1998), no. 1, 61-90.

22. J. Hu, Gromov-Witten invariants of blow-ups along points and curves, Math. Z. 233 (2000), no. 4, 709-739. MR 2001c:53115

23. V. A. Iskovskih, Fano threefolds. I, Izv. Akad. Nauk SSSR Ser. Mat. 41 (1977), no. 3, 516-562, 717. MR 80c: $14023 \mathrm{a}$

24. _ Fano threefolds. II, Izv. Akad. Nauk SSSR Ser. Mat. 42 (1978), no. 3, 506-549. MR 80c: $14023 \mathrm{~b}$

25. M. Kontsevich and Yuri I. Manin, Gromov-Witten classes, quantum cohomology, and enumerative geometry, Comm. Math. Phys. 164 (1994), no. 3, 525-562. MR 95i:14049

26. A. G. Kuznetsov, An exception set of vector bundles on the varieties $V_{22}$, Vestnik Moskov. Univ. Ser. I Mat. Mekh. (1996), no. 3, 41-44, 92. MR 97m:14040

27. Jun Li and Gang Tian, Virtual moduli cycles and Gromov-Witten invariants of algebraic varieties, J. Amer. Math. Soc. 11 (1998), no. 1, 119-174. MR 99d:14011

28. Shigefumi Mori and Shigeru Mukai, Classification of Fano 3-folds with $B_{2} \geq 2$, Manuscripta Math. 36 (1981/82), no. 2, 147-162. MR 83f:14032 
29. 느 Erratum to "classification of Fano 3-folds with $B_{2} \geq 2$ ", Manuscripta Math. 110 (2003), 407.

30. D. O. Orlov, Exceptional set of vector bundles on the variety $V_{5}$, Vestnik Moskov. Univ. Ser. I Mat. Mekh. (1991), no. 5, 69-71. MR 95f:14080

31. Z. Qin and Y. Ruan, Quantum cohomology of projective bundles over $\mathbb{P}^{n}$, Transactions of the Am. Math. Soc. 350 (1998), no. 9, 3615-3638.

32. Yongbin Ruan and Gang Tian, A mathematical theory of quantum cohomology, J. Differential Geom. 42 (1995), no. 2, 259-367. MR 96m:58033

33. Holger Spielberg, The Gromov-Witten invariants of symplectic toric manifolds, and their quantum cohomology ring, C. R. Acad. Sci. Paris Sér. I Math. 329 (1999), no. 8, 699-704. MR 2000j:14087

34. Michał Szurek and Jarosław A. Wiśniewski, Fano bundles of rank 2 on surfaces, Compositio Math. 76 (1990), no. 1-2, 295-305, Algebraic geometry (Berlin, 1988). MR 92e:14037

35. Keiichi Watanabe and Masayuki Watanabe, The classification of Fano 3-folds with torus embeddings, Tokyo J. Math. 5 (1982), no. 1, 37-48. MR 83m:14029

Dipartimento di Matematica "Ulisse Dini", Viale Morgagni 67A, 50134 Firenze, ITALY E-mail address: ciolli@math.unifi.it

URL: http://www.math.unifi.it/ ciolli 